\title{
Improved patient safety through reduced airway infection rates in a paediatric cystic fibrosis programme after a quality improvement effort to enhance infection prevention and control measures
}

\author{
Adrienne P Savant, ${ }^{1,2}$ Catherine O'Malley, ${ }^{3}$ Stacy Bichl, ${ }^{1}$ \\ Susanna A McColley ${ }^{1,2}$
}

${ }^{1}$ Division of Pulmonary Medicine, Department of Pediatrics, Ann \& Robert H. Lurie Children's Hospital of Chicago, Chicago, Illinois, USA ${ }^{2}$ Northwestern University Feinberg School of Medicine, Chicago, Illinois, USA ${ }^{3}$ Respiratory Department, Ann \& Robert H. Lurie Children's Hospital of Chicago, Chicago, Illinois, USA

\section{Correspondence to} Dr Adrienne Prestridge Savant, Division of Pulmonary Medicine, Department of Pediatrics, Ann \& Robert H. Lurie Children's Hospital of Chicago, $225 \mathrm{E}$ Chicago Ave Box \#43, Chicago, IL 60611, USA

asavant@|uriechildrens.org

Received 12 July 2013 Revised 24 October 2013 Accepted 4 December 2013

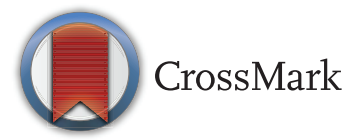

To cite: Savant AP, O'Malley C, Bichl S, et al. BMJ Qual Saf 2014;23: i73-i80.

\begin{abstract}
Objective To reduce the risk of pathogen transmission between patients with cystic fibrosis (CF) and decrease the rate of acquisition of new CF pathogens in our patients.

Design Using the Model for Improvement, we developed a new process for infection prevention and control in our outpatient CF clinics.

Setting Paediatric CF programme at Ann \& Robert H. Lurie Children's Hospital of Chicago; approximately 180 paediatric patients aged birth to 21 years.

Participants All paediatric patients enrolled in the Cystic Fibrosis Foundation Patient Data Registry at this institution.

Interventions Implemented contact precautions with all patients, regardless of respiratory tract culture results.
\end{abstract}

Measurement Respiratory tract culture rates of specific pathogens by quarter were compared prior to and after implementation.

Results Our percentage of patients with a positive respiratory tract culture for Pseudomonas aeruginosa dropped from $30 \%$ to $21 \%$ $(\mathrm{p}<0.0001)$ and for methicillin-resistant Staphylococcus aureus (MRSA) dropped from $10.8 \%$ to $8.7 \%(p=0.008)$.

Conclusions Use of contact precautions by all care providers, for all patients, regardless of respiratory tract culture results resulted in decreased $P$ aeruginosa and MRSA infection rates.

\section{INTRODUCTION}

Cystic fibrosis (CF) is a life-shortening illness characterised by chronic airway infection that leads to inflammation and destruction of the lungs, resulting in bronchiectasis and respiratory failure. An important safety concern for patients with $\mathrm{CF}$ is to limit the transmission of CF-specific bacteria between patients due to serious prognostic implications of specific respiratory tract infections. Pseudomonas aeruginosa infection is associated with worse pulmonary function, lower chest radiograph scores, poor growth, increased need for antibiotic therapy, greater likelihood of hospitalisations and increased mortality. ${ }^{1}{ }^{2}$ Furthermore, recent evidence demonstrates that the presence of methicillinresistant Staphylococcus aureus (MRSA) in respiratory cultures is harmful to patients as demonstrated by increased courses of intravenous antibiotics, worse baseline chest radiographs, decline in lung function and decreased survival. ${ }^{1}{ }^{3-5}$ Patients with MRSA or $P$ aeruginosa have a higher risk of death compared with patients without, and thus any measures to limit patient to patient transmission should be taken in order to prolong life. ${ }^{36}$

The clinical impact of acquisition of these infections, combined with the evidence for patient-to-patient transmission, ${ }^{2}$ 578 led to the development of specific national infection control guidelines for $\mathrm{CF}^{1}$ The sentinel pathogen in $\mathrm{CF}$ was Burkholderia cepacia complex (BCC) because of its high transmissibility and serious morbidity. ${ }^{9}$ In the early 1980 s, B cepacia emerged as a respiratory pathogen 
in CF. It quickly became apparent that this infection could lead to high fever, bacteraemia, progressive necrotising pneumonia and a rapid pulmonary decline, described as the 'cepacia syndrome', leading to death in $62-100 \%$ of infected patients. ${ }^{10}{ }^{11}$ In the early 1990 s, genetic testing methods provided proof that people with $\mathrm{CF}$ were transmitting $B$ cepacia to one another through social contact (ie, summer camps, exercise classes and parties). ${ }^{12-16}$ The evidence of patient-to-patient spread of $B$ cepacia caused considerable fear of an epidemic and led to dramatic changes in the infection control practices at CF centres worldwide. ${ }^{9} 12141718$ Although these changes were challenging, especially for people with CF who previously enjoyed a tight social network, strict infection control practices were effective in preventing outbreaks of $B$ cepacia. ${ }^{19}$

In 2007, at the Ann \& Robert H. Lurie Children's Hospital of Chicago, we began planning a quality improvement (QI) initiative at our CF centre to improve our infection prevention and control processes. At that time, we used contact isolation related to specific pathogens as outlined by the Cystic Fibrosis Foundation (CFF) Infection Control Guidelines, including contact precautions in CF patients infected with epidemiologically important CF pathogens, for example, BCC, MRSA or multidrug-resistant $P$ aeruginosa. This recommendation applied to both the inpatient and the outpatient settings. Despite the availability of evidence-based recommendations for infection control and the critical nature of this safety issue, fewer than $50 \%$ of healthcare workers, across all CF centres, adhered to these guidelines. ${ }^{1} 19$

At the Ann \& Robert H. Lurie Children's Hospital of Chicago, in 2007, the respiratory tract culture rates for MRSA in CF patients were lower than the national CF centre average, $15 \%$ as compared with $21.2 \%$. The infection rates for $P$ aeruginosa in $C F$ patients were similar to the national CF centre average, $42.1 \%$ as compared with $39.1 \%$.

The CFF recommends frequent respiratory tract (oropharyngeal swab or sputum) cultures in order to identify and treat patients with infection. However, using standard microbiology laboratory practices, identification of pathogens from an individual patient can take up to a week, and even then may not isolate $100 \%$ of CF pathogens in the airway of the patient at the time of the encounter. It is possible that a patient with previously negative cultures could come to clinic and spread infection. Isolation or cohorting based solely on historical respiratory tract culture results can risk transmission of a new, previously unidentified pathogen. Therefore, in order to improve the safety of our patients, we hypothesised that by using contact precautions with all patients by all healthcare workers, regardless of respiratory tract culture results, we could decrease the rate of transmission of CF pathogens and the acquisition of new bacteria in our patients.

\section{METHODS}

The CF Centre at the Ann \& Robert H. Lurie Children's Hospital of Chicago provides care to approximately 180 paediatric patients (age 021 years) with CF. Prior to 2007, our CF centre had an infection prevention and control policy in place for outpatients, which included immediate 'rooming' (placement within the examination room) of all patients to avoid patient-to-patient transmission and contact contamination in the waiting room. We did not segregate clinics based on respiratory tract culture results (ie, separate clinics for patients who grew $P$ aeruginosa, multidrug-resistant organism, etc.) or for newborns, as done in some other CF centres. Vital signs were performed in a common station in the hallway close to the exam rooms, without specific cleaning between patients. Patients routinely had surveillance oropharyngeal or sputum respiratory tract cultures obtained every 2-3 months, and any patient with respiratory tract cultures revealing a multidrug-resistant organism or MRSA had a flag placed on the chart to indicate the need for contact precautions. However, a consistent process for the use of this indicator was not systematic or routine.

A QI initiative was started in 2007 to prevent transmission of pathogens between CF patients. Many of the clinicians involved in the project had extensive practical experience in QI efforts through learning collaboratives sponsored by the CFF. We used the Model for Improvement ${ }^{20}$ as the framework for the initiative. A multidisciplinary QI team, including family and patient advisors, convened using the microsystems methodology ${ }^{21}$ to approach infection prevention and control. Respiratory tract culture results were tracked using a customised report from the CFF registry. Patients or their families had previously provided consent for registry participation.

The key strategy in our QI initiative was to implement contact precautions for all patients by all healthcare workers in the outpatient clinic, regardless of respiratory tract culture results. To accomplish this, we conducted several tests of change that included structural changes, changes in the process of care and education of providers and patients. (box 1). These changes were developed and tested via Plan-Do-Study-Act (PDSA) cycles, and all team members, including the nursing assistants and point of service staff, were responsible for these changes. All changes were instituted at our main hospital location and three satellite clinics with minor modifications to the process flow (but not the procedures) based on specific physical environment at each location.

There were many aspects of our QI initiative that supported our change to contact precautions for all patients, as outlined in box 1 , such as education of providers and patients, numerous structural changes to facilitate the ease of using contact precautions and significant new processes outlined for all team members. The specific keys to our success included 
Structural changes

- improving the signage for infection prevention and control measures to encourage the use of hand gel and mask by all patients when they entered the facility or were otherwise outside of the exam room;

- arranging the ready availability of hand gel and paediatric and adult-sized masks;

- arranging an adequate supply of gowns and gloves;

- abolishing the designated communal area for taking vital sign measurements and converting to in exam room vitals;

placing trashcans with foot pedals in the exam rooms to facilitate removal of protective gear in clinic exam room;

- placing individual stadiometers and scales in the exam room where possible;

- availability of alcohol wipes for cleaning stethoscopes after examining the patient for clinicians who did not wish to use disposable stethoscopes.

Process changes

- contact precautions for all patients: hand hygiene, gowning and gloving by all providers prior to entering the clinic exam room for all patients;

- requesting that all patients use hand gel and mask when entering the facility or when outside of the exam room, with reminders to patients from all care givers, from clinic check in onward;

- performing vital signs in the exam room;

- using disposable equipment, such as blood pressure cuffs and pulse oximeters;

- cleaning of reusable equipment used for vital measurement between patients;

- cleaning of exam room after every patient.

Education of providers

- the respiratory therapist and the infection control team determined how best to 'thoroughly' clean the exam room between patients; how many wipes to use, what items in the room needed wiping and in what order;

- the creating of a step-by-step procedure and the training of the nursing assistants on the process for thoroughly cleaning the exam rooms;

- at a CF staff meeting, the physicians demonstrated how to efficiently and correctly gown and glove;

- all team members are responsible to promote and train everyone in the clinic on the new processes, and this responsibility is continuous.

Education of patients and families

- development of a survey to identify gaps in knowledge and understanding of CF infection control and pulmonary health;

- a letter to every family informing and preparing them for the initiation of new infection control processes, explaining both the clinicians and families role;

- an article in the CF newsletter sent to families describing the purpose and process of the new infection control changes;

- age-appropriate educational material on germs and infection prevention and control measures distributed to the children in clinic;

- educational information to address the specific knowledge gaps assessed from the survey was presented at an annual family education day.

continued re-enforcement of the 'no-waiting' room policy, education of patients and families about the risk of patient-to-patient transmission of bacteria, cleaning rooms thoroughly and contact precautions for all patients. Prior to the implementation, all parents received our centre's newsletter and a personal letter outlining the importance of good infection control practices, which was again reinforced in clinic. When our patients come for appointments, they are taken immediately from registration and placed in a clinic room, where all care is done, from vital signs to visits with all clinicians, thus avoiding a common waiting room. Only pulmonary function tests (PFTs) are done in another room, where the PFT staff use contact precautions. In the clinic rooms, all clinicians-nursing assistants, social workers, respiratory therapists, nutritionists, nurses and physiciansuse contact precautions when entering the room. Finally, we have a thorough process for cleaning the clinic and pulmonary function rooms between patients, which includes wiping all surfaces with bactericidal wipes.

The new infection control process was implemented with the paediatric population in October 2007. The same process was implemented in our adult clinic; however, adult data are not analysed or shown here. Our specific outcome measure was the percentage of patients with a specific organism isolated on respiratory tract culture, by quarter, using data extracted from the CFF Patient Data Registry. The registry 
currently tracks the health outcomes of approximately 23000 patients in 115 CF centres. The data analysis from the registry includes centre-specific data as compared with other centres as well as progress over time for rates of infections. The CFF Patient Registry has received approval from our Institutional Review Board. At the end of each quarter, the percentage of patients with a positive respiratory tract culture for a specific pathogen, defined as number of patients with a pathogen in one or more respiratory tract culture specimens divided by total number of patients who had cultures (thus each positive respiratory tract culture for a specific pathogen only counted once per patient). A comparison between proportions was done using Student $\mathrm{t}$ tests.

\section{RESULTS}

We evaluated data from 2005 to 2012 in order to have a baseline of our infection rates. Data collection was done on a quarterly basis from the first quarter of 2005 through the fourth quarter of 2007. Contact precautions for all patients, regardless of respiratory tract culture results, were started late in quarter 4 of 2007. Data up to the end of 2012 are reported here. Baseline and ongoing demographics for our programme are presented in table 1 . In 2005 at the start of our baseline monitoring period, there were 127 total patients with cultures in the year, 59\% $(n=75)$ of those patients continue to be followed in our programme in 2012, while 35\% $(n=45)$ have transferred to other programmes, including transition to our adult CF programme, and 6\% $(\mathrm{n}=7)$ have died. In 2005,8 of the 127 patients were new to our centre, half being new diagnoses and half transferring to our centre.

During this time, we saw on average 128 patients per quarter; however, the number of patients ranged in the baseline period from 104 to 121 patients per quarter, while in the postintervention period we ranged from 117 to 155 patients per quarter. Patients had between one and four respiratory tract cultures per quarter. We obtained a mean of 169 respiratory tract cultures per quarter over the study period (inpatient and outpatient), ranging from 104 to 207 respiratory tract cultures per quarter in baseline period and 173-206 respiratory tract cultures per quarter postintervention.

During this time frame, patients were added to our clinic as transfers or new diagnoses. For transfers to our centre, we examined the number of patients that came to our centre with either P aeruginosa or MRSA. We had 52 transfers from 2005 to 2012 with 27\% $(\mathrm{n}=14)$ having $P$ aeruginosa on transfer and $12 \%$ $(n=6)$ having MRSA on transfer.

The percentage of patients cultured each quarter with positive respiratory tract cultures for $P$ aeruginosa and MRSA significantly declined when comparing baseline to postintervention rates. (figure 1) Prior to the new process, our centre had a mean of 29.79\% (range 38.74-22.94\%) of patients per quarter with a respiratory tract culture positive for $P$ aeruginosa compared with after initiation with a mean of $21.78 \%$ (range $31.09-12.95 \%)(\mathrm{p}<0.0001)$. MRSA had a similar decline from a mean of $10.76 \%$ (range $12.5-7.34 \%$ ) to $8.68 \%$ (range 12.78-5.38\%) $(\mathrm{p}=0.008)$.

Table 1 Demographics for the patients from Ann \& Robert H. Lurie Children's Hospital of Chicago (Lurie Children's)

\begin{tabular}{|c|c|c|c|c|c|c|c|c|}
\hline & 2005 & 2006 & 2007 & 2008 & 2009 & 2010 & 2011 & 2012 \\
\hline Number of patients & 129 & 126 & 144 & 148 & 161 & 166 & 177 & 175 \\
\hline Age, mean (SD) & $9.7(5.9)$ & $10.0(5.4)$ & $10.0(5.6)$ & $10.5(5.6)$ & $10.4(6.0)$ & $10.0(5.9)$ & $10.1(6.0)$ & $10.6(5.7)$ \\
\hline$\%$ male/\%female & $47 / 53$ & $45 / 55$ & $45 / 55$ & $45 / 55$ & $45 / 55$ & $46 / 54$ & $50 / 50$ & $49 / 51$ \\
\hline \multicolumn{9}{|l|}{ Ethnicity (\%) } \\
\hline Caucasian & 94.6 & 94.4 & 94.4 & 94.6 & 95.0 & 94.0 & 94.4 & 94.9 \\
\hline African American/Black & 4.7 & 4.0 & 4.2 & 4.1 & 3.7 & 4.2 & 4.0 & 3.4 \\
\hline Asian & 1.6 & 1.6 & 1.4 & 1.4 & 1.2 & 1.2 & 1.1 & 1.1 \\
\hline Other & 0 & 0 & 0 & 0 & 0 & 0.6 & 0.6 & 1.2 \\
\hline \multicolumn{9}{|l|}{ Genotype (\%) } \\
\hline Delta F508 homozygous & 47.3 & 47.6 & 46.5 & 46.6 & 47.2 & 45.2 & 41.8 & 40.0 \\
\hline Delta F508 heterozygote & 35.7 & 34.9 & 37.5 & 37.8 & 35.4 & 36.7 & 38.4 & 41.1 \\
\hline Other mutations (no delta F508) & 15.5 & 16.7 & 16.0 & 15.5 & 17.4 & 18.1 & 19.2 & 18.9 \\
\hline No genotype data & 1.6 & 0.8 & 0 & 0 & 0 & 0 & 0.6 & 0 \\
\hline Median BMI percentile $2-20$ years & 53 & 55.5 & 53.1 & 50.5 & 55.7 & 58.8 & 64.5 & 60.4 \\
\hline Percent on pancreatic enzymes (\%) & 89.1 & 87.3 & 88.2 & 88.5 & 87.6 & 84.3 & 81.9 & 80.0 \\
\hline $\begin{array}{l}\text { FEV1 }(\% \text { predicted }) \\
6-17 \text { years }\end{array}$ & 88 & 91 & 92 & 92 & 92 & 95 & 93 & 93 \\
\hline $\begin{array}{l}\text { Inhaled Tobramycin use (>6 years old with } \\
\text { Pseudomonas) }\end{array}$ & 89.9 & 74.2 & 76.4 & 73.7 & 77.5 & 76.3 & 73.8 & 71.7 \\
\hline $1+$ pulmonary exacerbations per year $(\%)<18$ years & 31.9 & 25.6 & 29.2 & 23.7 & 24.8 & 23.7 & 16.4 & 25.5 \\
\hline
\end{tabular}




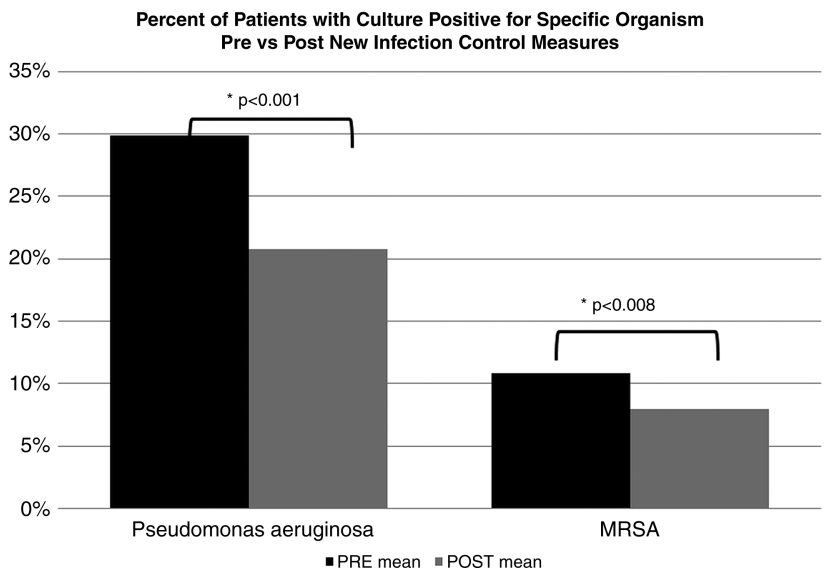

Figure 1 Percentage of patients per quarter with a positive respiratory tract culture for specific pathogens. Prebars (black) are before implementation of our intervention of placing all patients in contact isolation, regardless of respiratory tract culture results (contact precautions). Postbars (grey) are after implementation of new infection control and prevention measures.

Using separately collected data from the Cystic Fibrosis Patient Registry (figure 2), the percentage of all patients in the nation with culture positive for $P$ aeruginosa or MRSA was compared with our centre (figure 2). The national averages of $P$ aeruginosa (in patients $\leq 18$ years of age) at $\mathrm{CF}$ centres in the
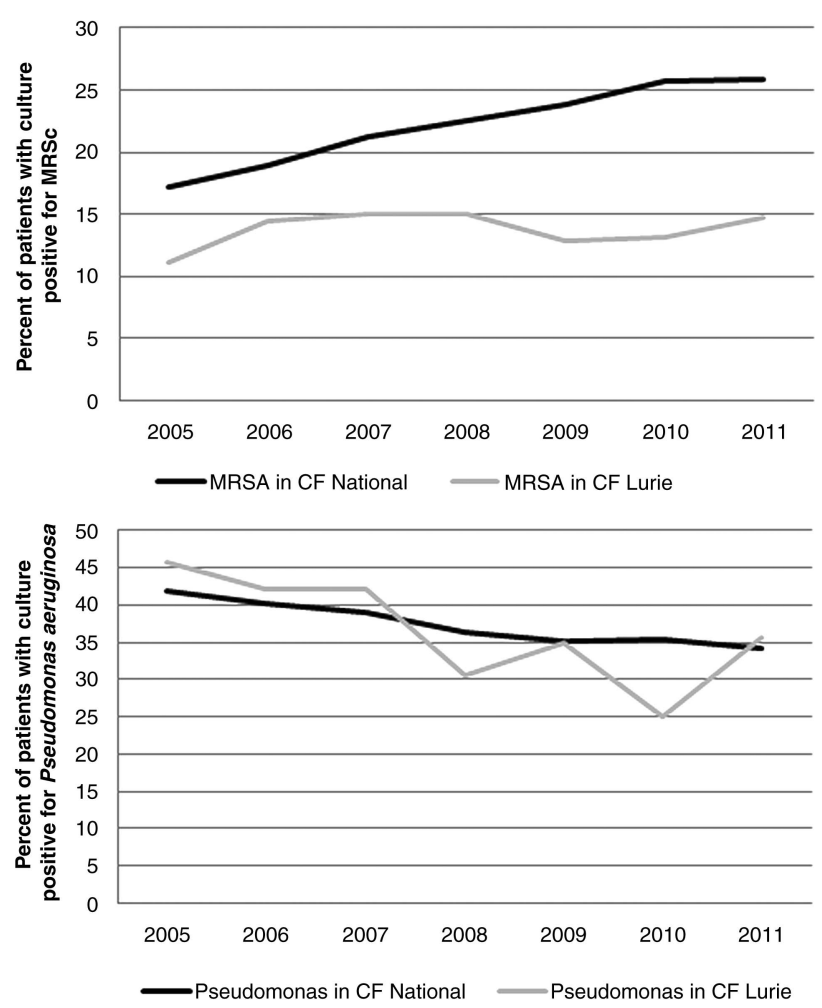

Figure 2 Percentage of patients per year with positive respiratory tract culture for specific pathogens. Top panel shows comparison of Lurie Children's percentage of positive patients for methicillin-resistant Staphylococcus aureus compared with the national cystic fibrosis (CF) population as tracked through the Cystic Fibrosis Foundation Patient Registry. Lower panel shows the same for Pseudomonas aeruginosa. same time frame went from an average of $40.4 \%$ (2005-2007) to $35.2 \%(2008-2011)$, while our rates declined from an average of $43.3 \%$ to $31.5 \% .^{22-28}$ In the same time frame, MRSA rates rose from an average of $19.1 \%$ (2005-2007) to $24.5 \%$ (20082011), while our rates remained the same, an average of $13.5 \%$ (2005-2007) to $13.95 \%$ (2008-2011), as tracked by the Cystic Fibrosis Patient Registry. ${ }^{22-28}$

\section{DISCUSSION}

The implementation of a new infection prevention process in our clinic led to statistically significant decreases in the number of patients with respiratory tract cultures positive for $P$ aeruginosa and MRSA, two specific organisms known to be associated with increased morbidity and mortality that are transmitted through patient-to-patient contact. Our centre did not have a 'sentinel' microorganism leading us to increase infection control measures. Rather, the incentive to optimise our infection prevention processes stemmed from an acknowledgement that any patient could potentially be harbouring an infectious pathogen and a desire to improve CF patient safety by minimising the risk of patient-to-patient transmission of these organisms. This is the first report, to our knowledge, of decreased infection rates following the implementation of contact precautions for all patients in an outpatient CF clinic, regardless of respiratory tract culture results.

The most important safety issue in the CF community is infection control. To date, evidence of patient-to-patient transmission of organisms among patients with CF continues to increase, and with devastating outcomes. In a recently published report of an outbreak of Mycobacteria abscessus ss. massiliense, five patients with CF were infected with indistinguishable isolates resulting in the death of three. An investigation of the outbreak determined that the only 
shared space between these patients was overlapping CF clinic days. ${ }^{29}$ Similarly, whole genome sequencing in a centre in the UK demonstrated patient-to-patient transmission of $M$ abscessus ss. massiliense, lending further support to providing contact precautions for all patients, since we are discovering that new pathogenic organisms can be spread from person to person. ${ }^{30} 31$ Another recently published outbreak is the Liverpool epidemic strain of $P$ aeruginosa. Molecular genotyping identified this as a common strain infecting patients with CF both in Canada and the UK, which is evidence of cross-contamination. People infected with the Liverpool strain had a greater rate of death or lung transplantation than those people infected with a unique strain of $P$ aeruginosa. ${ }^{2}$ There has also been a report of a multidrug-resistant $P$ aeruginosa (Houston-1), where patients had an increased risk of acquisition if they had been hospitalised. Infection control improvements led to reduced incidence of this strain. ${ }^{32}$ The most important lessons learned from these outbreaks is that the evidence of patient-to-patient transmission of pathogens between people with CF is certain, and that infection prevention and control practices play a vital role in limiting or halting further transmission. ${ }^{29}{ }^{33}$ The emergence of new evidence of patient-to-patient transmission of organisms, not previously known to have been spread through this mechanism, makes it crucial that we work to protect our patients from both known and unknown pathogens.

In a survey of CF care centres in the USA shortly after publication of the CFF Infection Control Guidelines, ${ }^{1}$ only $58 \%$ of respondents had an outpatient policy written that recommended contact precautions for BCC, multidrug-resistant organisms or MRSA. ${ }^{34}$ This differs from a similar survey in Europe, where $68-93 \%$ of sites separated outpatients based on these organisms. ${ }^{35}$ In both surveys, there was a lack of a written policy in $23-35^{34} \%$ of the respondents. ${ }^{34} 35$ The European survey, however, had a low response rate $(32 \%)$ compared with the US survey (85\%) and may account for the differences in apparent implementation of the guidelines. ${ }^{3435}$ Less than half of the respondents had outpatient policies discouraging socialisation between individuals with $\mathrm{CF}$ or to remain more than three feet from others with $\mathrm{CF}^{34}$ Many other centres have described their efforts at segregation based on respiratory tract culture results; ${ }^{36-40}$ however, to our knowledge, this is the first publication describing the use of contact precautions for all patients, regardless of respiratory tract culture results.

Common barriers to implementation of infection control guidelines in any healthcare setting relate to knowledge (familiarity and awareness), attitudes (selfefficacy and outcome expectancy) and behaviour (patient factors and environmental factors). ${ }^{41}$ Physicians express concern about the social isolation of patients as a significant barrier. Additionally 31-47\% of healthcare providers demonstrated a lack of awareness and were unfamiliar with specific guidelines for $\mathrm{CF}$ infection control. ${ }^{19}$ In CF, lack of time and lack of support staff to clean rooms was cited as a barrier to infection control in $24 \%$ and $27 \%$ of CF providers, respectively. ${ }^{9}$ A survey specific to $\mathrm{CF}$ and administered to patients, families and care givers revealed that only $60 \%$ were aware of infection control guidelines and only $30 \%$ of patients and families had discussed the guidelines with a CF care team member. ${ }^{19}{ }^{42}$ Despite this low rate of awareness, the survey revealed there was a high rate of confidence (self-efficacy) and positive health effect (outcome expectancy) expressed by patients and families in regards to specific infection control practices. ${ }^{42}$ In a similar analysis in Australia, $85 \%$ of parents and $63 \%$ of patients over age 12 were positive about segregation measures as part of infection control. ${ }^{43}$ Access to the guidelines did not improve adherence, signalling the inadequacy of education and familiarity to changing behaviour. ${ }^{19}$

Important lessons can be garnered from this work, both within the CF community and beyond. Specifically, any change can take a long time and requires continued surveillance. Our discussion and planning for this process lasted 9 months before we implemented the changes. We have continued to track our data to ensure that we are sustaining our change. For the infection control community, dedicated processes to an infection can halt the further spread and ensure that all clinicians abide by the process.

Keys to our QI initiative in overcoming barriers were designated CF clinician champions and a strong confidence that the changes would make a difference. Specifically, to overcome the barrier of lack of knowledge, we ensured all staff had access to the guidelines and had numerous staff educational sessions, while patients and families were provided educational materials in the form of a newsletter, letter to family and in-clinic verbal explanation. Our staff was highly confident that the changes we implemented would result in a positive outcome for the safety of our patients, and this positive attitude was reflected in our communication with parents. Although we expected resistance from patients and families, on the whole our changes were viewed positively (although not formally assessed). Our team did encounter barriers; most critical to a QI project was the inability to evaluate small tests of change since we felt it was impractical to do only parts of our new cleaning and isolation procedures.

This study is limited by the fact that it was done as a QI initiative in which cross-sectional data were used. This cohort was not followed prospectively, and there were no matched controls. Finally, we implemented a practice of prescribing eradication therapy for initial $P$ aeruginosa infection during this time period, which would be expected to reduce the prevalence of $P$ aeruginosa, while not affecting the rates of other pathogens. There was no specific practice change for the 
treatment of MRSA in CF to account for the decline in patients with a positive culture for this organism. Within our hospital, the only change for MRSA during this time frame occurred in our intensive care setting, where our patients rarely are placed. In general, hospitals track rates of MRSA found from a sterile site, categorising the infections as healthcare associated, community onset, hospital onset or community associated, making a comparison to $\mathrm{CF}$ respiratory tract cultures less direct. However, during a similar time frame, from 2005 to 2010 , the rates of invasive MRSA in children (without $\mathrm{CF}$ ) saw no significant reductions despite the trends in adult healthcare that show declines in overall MRSA rates from 2005 to $2011^{44} 45$

We have continued to analyse the data on a quarterly basis and have expanded our tracking to include additional organisms. Periodic evaluation and review of our practices related to infection prevention have been made, and these practices altered to accommodate changes to the clinical environment or to incorporate new evidence. Regular reinforcement of these practices with all healthcare providers (both those seasoned and new to the centre) is necessary to maintain the improvements made in decreasing pathogen rates.

The outcomes of this improvement initiative are particularly important to the CF community in light of recent reports of patient-to-patient spread leading to devastating outcomes ${ }^{29} 33$ As the CF microbiome changes over time, new organisms and changes in virulence patterns occur, heightening the need for improved safety for all patients with CF related to infection prevention and control. We believe this 'zero tolerance' policy for patient-to-patient pathogen transmission could be considered in other patient populations.

In conclusion, this study demonstrates the effectiveness of improved infection prevention practices through the use of contact precautions for all those seen in the outpatient setting regardless of respiratory track culture for reducing the acquisition and spread of potentially life-shortening infection in CF.

Acknowledgements We would like to thank all members of our cystic fibrosis team for their tireless attention to infection prevention and control. More importantly, we would like to thank all of our patients and families for helping us to deliver better care on a daily basis.

Contributors All authors made the following contributions: substantial contributions to the conception or design of the work; or the acquisition, analysis or interpretation of data for the work; drafted the work and revised it critically for important intellectual content; final approval of the version to be published; and agreed to be accountable for all aspects of the work in ensuring that questions related to the accuracy or integrity of any part of the work are appropriately investigated and resolved

Funding None.

Competing interests None.

Ethics approval Institutional Review Board.

Provenance and peer review Not commissioned; externally peer reviewed.

\section{REFERENCES}

1 Saiman L, Siegel J. Infection control recommendations for patients with cystic fibrosis: Microbiology, important pathogens, and infection control practices to prevent patient-to-patient transmission. Am J Infect Control 2003;31(3 Suppl):S1-62.

2 Aaron SD, Vandemheen KL, Ramotar K, et al. Infection with transmissible strains of Pseudomonas aeruginosa and clinical outcomes in adults with cystic fibrosis. JAMA 2010;304:2145-53.

3 Dasenbrook EC, Checkley W, Merlo CA, et al. Association between respiratory tract methicillin-resistant Staphylococcus aureus and survival in cystic fibrosis. JAMA 2010;303:2386-92.

4 Sawicki GS, Rasouliyan L, Ren CL. The impact of MRSA on lung function in patients with cystic fibrosis. Am J Respir Crit Care Med 2009;179:734-5; author reply 5.

5 Vanderhelst E, De Meirleir L, Verbanck S, et al. Prevalence and impact on FEV(1) decline of chronic methicillin-resistant Staphylococcus aureus (MRSA) colonization in patients with cystic fibrosis. A single-center, case control study of 165 patients. J Cyst Fibros 2012;11:2-7.

6 Emerson J, Rosenfeld M, McNamara S, et al. Pseudomonas aeruginosa and other predictors of mortality and morbidity in young children with cystic fibrosis. Pediatr Pulmonol 2002;34:91-100.

7 Fothergill JL, Walshaw MJ, Winstanley C. Transmissible strains of Pseudomonas aeruginosa in cystic fibrosis lung infections. Eur Respir J 2012;40:227-38.

8 Al-Aloul M, Crawley J, Winstanley C, et al. Increased morbidity associated with chronic infection by an epidemic Pseudomonas aeruginosa strain in CF patients. Thorax 2004;59:334-6.

9 Saiman L, Siegel J. Infection control in cystic fibrosis. Clin Microbiol Rev 2004;17:57-71.

10 Isles A, Maclusky I, Corey M, et al. Pseudomonas cepacia infection in cystic fibrosis: an emerging problem. $J$ Pediatr 1984;104:206-10.

11 Tablan OC, Martone WJ, Doershuk CF, et al. Colonization of the respiratory tract with Pseudomonas cepacia in cystic fibrosis. Risk factors and outcomes. Chest 1987;91:527-32.

12 Zuckerman JB, Seder DB. Infection control practice in cystic fibrosis centers. Clin Chest Med 2007;28:381-404.

13 Thomassen MJ, Demko CA, Doershuk CF, et al. Pseudomonas cepacia: decrease in colonization in patients with cystic fibrosis. Am Rev Respir Dis 1986;134:669-71.

14 LiPuma JJ, Dasen SE, Nielson DW, et al. Person-to-person transmission of Pseudomonas cepacia between patients with cystic fibrosis. Lancet 1990;336:1094-6.

15 Pegues DA, Carson LA, Tablan OC, et al. Acquisition of Pseudomonas cepacia at summer camps for patients with cystic fibrosis. Summer Camp Study Group. J Pediatr 1994;124(5 Pt 1):694-702.

16 Johnson WM, Tyler SD, Rozee KR. Linkage analysis of geographic and clinical clusters in Pseudomonas cepacia infections by multilocus enzyme electrophoresis and ribotyping. J Clin Microbiol 1994;32:924-30.

17 Govan JR, Brown PH, Maddison J, et al. Evidence for transmission of Pseudomonas cepacia by social contact in cystic fibrosis. Lancet 1993;342:15-19.

18 Pseudomonas cepacia at summer camps for persons with cystic fibrosis. MMWR Morb Mortal Wkly Rep 1993;42:456-9.

19 Garber E, Desai M, Zhou J, et al. Barriers to adherence to cystic fibrosis infection control guidelines. Pediatr Pulmonol 2008;43:900-7. 
20 Langley GJ. The improvement guide: a practical approach to enhancing organizational performance. 2nd ed. San Francisco: Jossey-Bass, 2009.

21 Godfrey MM, Nelson EC, Wasson JH, et al. Microsystems in health care: Part 3. Planning patient-centered services. Jt Comm J Qual Saf 2003;29:159-70.

22 Cystic Fibrosis Foundation Patient Registry. 2005.

23 Cystic Fibrosis Foundation Patient Registry. 2006.

24 Cystic Fibrosis Foundation Patient Registry. 2007.

25 Cystic Fibrosis Foundation Patient Registry. 2008.

26 Cystic Fibrosis Foundation Patient Registry. 2009.

27 Cystic Fibrosis Foundation Patient Registry. 2010.

28 Cystic Fibrosis Foundation Patient Registry. 2011.

29 Aitken ML, Limaye A, Pottinger P, et al. Respiratory outbreak of Mycobacterium abscessus subspecies massiliense in a lung transplant and cystic fibrosis center. Am J Respir Crit Care Med 2012;185:231-2.

30 Bryant JM, Grogono DM, Parkhill J, et al. Transmission of M abscessus in patients with cystic fibrosis-Authors' reply. Lancet 2013;382:504.

31 Bryant JM, Grogono DM, Greaves D, et al. Whole-genome sequencing to identify transmission of Mycobacterium abscessus between patients with cystic fibrosis: a retrospective cohort study. Lancet 2013;381:1551-60.

32 Luna RA, Millecker LA, Webb CR, et al. Molecular epidemiological surveillance of multidrug-resistant Pseudomonas aeruginosa isolates in a pediatric population of patients with cystic fibrosis and determination of risk factors for infection with the Houston-1 strain. J Clin Microbiol 2013;51:1237-40.

33 Ashish A, Shaw M, Winstanley C, et al. Halting the spread of epidemic pseudomonas aeruginosa in an adult cystic fibrosis centre: a prospective cohort study. JRSM Short Rep 2013;4:1.

34 Zhou J, Garber E, Saiman L. Survey of infection control policies for patients with cystic fibrosis in the United States. Am J Infect Control 2008;36:220-2.
35 Elborn JS, Hodson M, Bertram C. Implementation of European standards of care for cystic fibrosis--control and treatment of infection. J Cyst Fibros 2009;8:211-17.

36 Festini F, Buzzetti R, Bassi C, et al. Isolation measures for prevention of infection with respiratory pathogens in cystic fibrosis: a systematic review. J Hosp Infect 2006;64:1-6.

37 Doe SJ, McSorley A, Isalska B, et al. Patient segregation and aggressive antibiotic eradication therapy can control methicillin-resistant Staphylococcus aureus at large cystic fibrosis centres. J Cyst Fibros 2010;9:104-9.

38 Griffiths AL, Jamsen K, Carlin JB, et al. Effects of segregation on an epidemic Pseudomonas aeruginosa strain in a cystic fibrosis clinic. Am J Respir Crit Care Med 2005;171:1020-5.

39 Griffiths AL, Wurzel DF, Robinson PJ, et al. Australian epidemic strain pseudomonas (AES-1) declines further in a cohort segregated cystic fibrosis clinic. J Cyst Fibros 2012;11:49-52.

40 Wiehlmann L, Cramer N, Ulrich J, et al. Effective prevention of Pseudomonas aeruginosa cross-infection at a cystic fibrosis centre-results of a 10-year prospective study. Int J Med Microbiol 2012;302:69-77.

41 Cabana MD, Rand CS, Powe NR, et al. Why don't physicians follow clinical practice guidelines? A framework for improvement. JAMA 1999;282:1458-65.

42 Miroballi Y, Garber E, Jia H, et al. Infection control knowledge, attitudes, and practices among cystic fibrosis patients and their families. Pediatr Pulmonol 2012;47:144-52.

43 Griffiths AL, Armstrong D, Carzino R, et al. Cystic fibrosis patients and families support cross-infection measures. Eur Respir J 2004;24:449-52.

44 Iwamoto $\mathrm{M}, \mathrm{Mu} \mathrm{Y}$, Lynfield $\mathrm{R}$, et al. Trends in invasive Methicillin-Resistant staphylococcus aureus infections. Pediatrics. 2013;132:e817-e24.

45 Dantes R, Mu Y, Belflower R, et al. National burden of invasive Methicillin-Resistant Staphylococcus aureus infections, United States, 2011. JAMA Intern Med 2013;173:1970-8. 